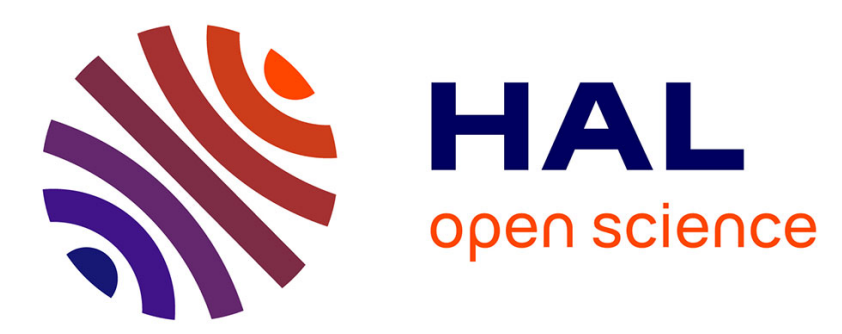

\title{
A mathematical model for dorsal closure
}

Luís Almeida, Patrizia Bagnerini, Abderrahmane Habbal, Stéphane Noselli, Fanny Serman

\section{To cite this version:}

Luís Almeida, Patrizia Bagnerini, Abderrahmane Habbal, Stéphane Noselli, Fanny Serman. A mathematical model for dorsal closure. Journal of Theoretical Biology, 2010, 268 (1), pp.105. 10.1016/j.jtbi.2010.09.029 . hal-00642433

\section{HAL Id: hal-00642433 https://hal.science/hal-00642433}

Submitted on 18 Nov 2011

HAL is a multi-disciplinary open access archive for the deposit and dissemination of scientific research documents, whether they are published or not. The documents may come from teaching and research institutions in France or abroad, or from public or private research centers.
L'archive ouverte pluridisciplinaire HAL, est destinée au dépôt et à la diffusion de documents scientifiques de niveau recherche, publiés ou non, émanant des établissements d'enseignement et de recherche français ou étrangers, des laboratoires publics ou privés. 


\title{
A Mathematical Model for Dorsal Closure
}

\author{
Luís Almeida ${ }^{1,4}$, Patrizia Bagnerini ${ }^{2}$, Abderrahmane Habbal ${ }^{1}$, Stéphane \\ Noselli $^{3}$ and Fanny Serman ${ }^{1,3}$ \\ ${ }^{1}$ Laboratoire J.A. Dieudonné \\ Université de Nice \\ UMR 6621 CNRS \\ Parc Valrose \\ F-06108 NICE Cédex 02, FRANCE \\ ${ }^{2}$ DIPTEM \\ Università degli Studi di Genova \\ P.le Kennedy-Pad D \\ 16129 Genova, ITALY \\ ${ }^{3}$ Institute of Developmental Biology \&3 Cancer IBDC-Nice \\ Université de Nice \\ UMR 6543 CNRS \\ Parc Valrose \\ F-06108 NICE Cédex 02, FRANCE \\ ${ }^{4}$ Corresponding author: Luis.Almeida@biopark-archamps.org, Current adress: \\ TIMC-IMAG, UJF UMR 5525 du CNRS, Bat. Le Forum, BioPark d'Archamps, \\ F-74160 Archamps, France
}

\begin{abstract}
During embryogenesis, Drosophila embryos undergo epithelial folding and unfolding, which leads to a hole in the dorsal epidermis, transiently covered by an extraembryonic tissue called the amnioserosa. Dorsal Closure (DC) consists of the migration of lateral epidermis towards the midline, covering the amnioserosa. It has been extensively studied since numerous physical mechanisms and signaling pathways present in DC are conserved in other morphogenetic events and wound healing in many other species (including vertebrates).
\end{abstract}


We present here a simple mathematical model for DC that involves a reduced number of parameters directly linked to the intensity of the forces in presence and which is applicable to a wide range of geometries of the Leading Edge (LE). This model is a natural generalization of the very interesting model proposed in Hutson, Tokutake, Chang, Bloor, Venakides, Kiehart, and Edwards (2003). Being based on an Ordinary Differential Equation (ODE) approach, the previous model had the advantage of being even simpler, but this restricted significantly the variety of geometries that could be considered and thus the number of modified dorsal closures that could be studied.

A Partial Differential Equation (PDE) approach, as the one developed here, allows considering much more general situations that show up in genetically or physically perturbed embryos and whose study will be essential for a proper understanding of the different components of the DC process. Even for native embryos, our model has the advantage of being applicable since the early stages of DC when there is no antero-posterior symmetry (approximately verified only in the late phases of DC).

We validate our model in a native setting and also test it further in embryos where the zipping force is perturbed through the expression of Spastin (a microtubule severing protein). We obtain variations of the force coefficients that are consistent with what was previously described for this setting.

Keywords: forces in embryogenesis, biomechanics, PDE models, tissue movement, drosophila, embryonic development, epithelia, actin cable, zipping, parameter optimization

\section{Introduction}

In this work we present a mathematical model of Dorsal Closure (DC) in Drosophila embryos which is a widely used biological model system for morphogenesis and wound healing. DC has been extensively studied as a paradigm of epithelial fusion events. In fact, a significant number of physical mechanisms and signaling pathways present in DC are conserved in many other morphogenetic and tissue repair events not only in Drosophila, but also in many other organisms (including vertebrates).

DC consists of the convergence of lateral epidermal cells towards the midline covering the amnioserosa in a couple of hours (see figure 1 and movie DCnative.mov). As it progresses, the left and right margins of the Leading Edge (LE) are progressively knitted to each other in a zipping process and 

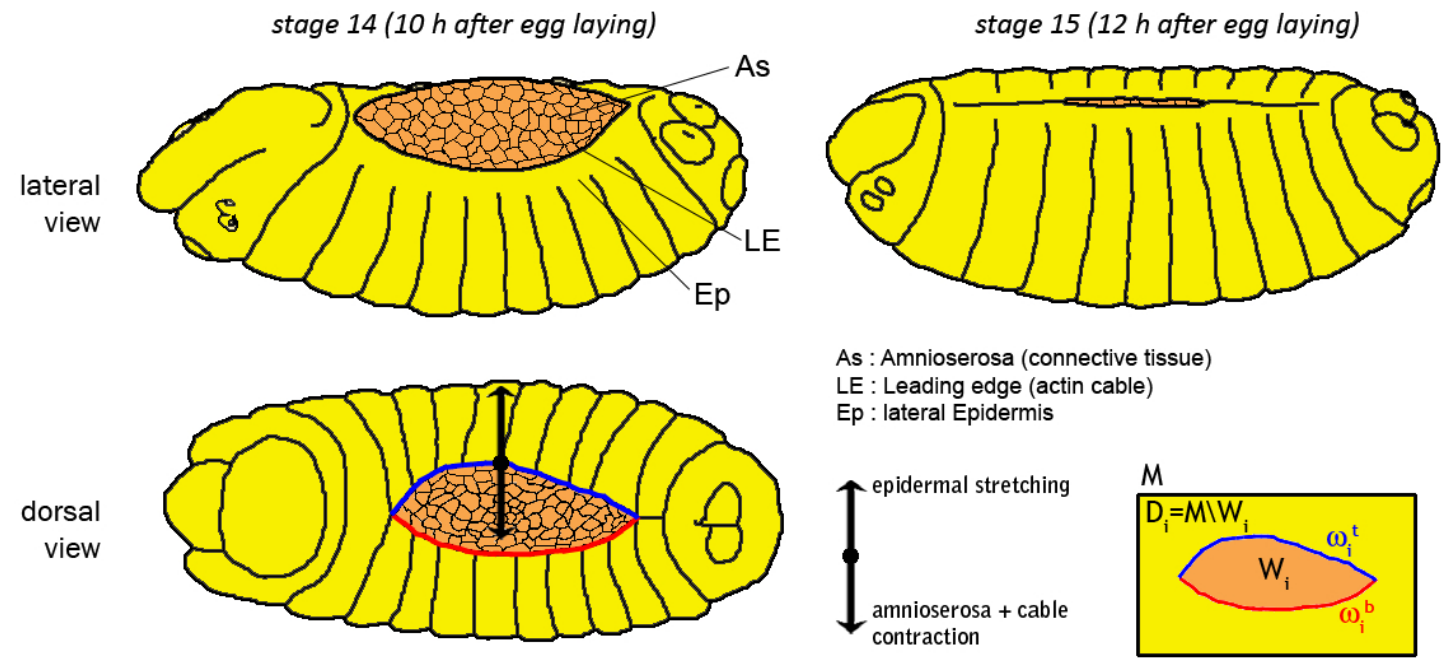

Figure 1: Scheme of tissues and forces implicated in dorsal closure in Drosophila embryos. The first row shows a dorso-lateral drawing of the drosophila embryo at stages 14 and 15. The second row shows a dorsal view of a stage 14 embryo and a representation of some of the forces involved in the closure. Lines indicate the two tissues involved (the amnioserosa consisting of thin and large polygonal epithelial cells in brown, the epidermis consisting of columnar epithelial cells in yellow) and in-between the Leading Edge (LE). In the last diagram we distinguish the LE's top margin $\omega_{i}^{t}$ (in blue) and bottom one $\omega_{i}^{b}$ (in red).

eventually seal the hole leaving no traces of its prior existence. In spite of the fact that the lateral epidermis has to encompass an increasing amount of area, DC does not involve any cell division but only a coordinated reorganization and contraction of the actomyosin cytoskeleton in different populations of epithelial cells.

The cells in the dorsal-most row of the lateral epidermis are called the leading edge cells - these cells form the boundary of the lateral epidermis and they accumulate filamentous actin and myosin II (a motor protein) at their dorsal-most edge to form a contractile actomyosin cable. Such intercellular cable elements are anchored into adherens junctions between neighboring cells (which are locally reinforced for this purpose) and are thus all connected forming a supra-cellular acto-myosin cable that encircles the area covered by the Amnioserosa. This cable structure is continuously put under tension 
by the myosin molecular motors making the actin filaments slide relative to each other thus producing a global contraction effect that helps closing the epithelial hole (since this is a dynamic process, new tension can be created by the molecular motors and the local cable tension does not necessarily decrease as the contraction proceeds). The position of this cable, at the boundary between the epidermis and the amnioserosa defines the Leading Edge (LE).

The hole is roughly shaped like an ellipse with major axis along the dorsal midline of the organism - which is along the anterior-posterior (AP) axis of the embryo. By convention, we will always display our images with the anterior on the left, the posterior on the right and a horizontal AP axis. The anterior and posterior ends of the hole are called the canthi - the LE has a rather singular geometry at these points. As indicated in figure 1, the canthi separate the LE (also denoted $\omega_{i}$ ) into two halves - the top margin, denoted $\omega_{i}^{t}$, and the bottom margin, $\omega_{i}^{b}$ (in fact, in the original geometry of the embryo, these correspond to the right and left margins, respectively).

In (Kiehart, Galbraith, Edwards, Rickoll, and Montague (2000)) DC was described as involving the following forces: 1) resistive tension from the stretched epidermis, 2) actin cable tension 3) amnioserosa contraction and 4) zipping.

The zipping force comes from the fact that LE cells extend actin protrusions, called filopodia (or lamellipodia when they start having a twodimensional structure in the late stages of zipping), that intertwine near the canthi drawing the two margins towards each other and knitting them - the canthi advance towards the middle of the epithelial hole occupied by the Amnioserosa a bit like zippers (see movie zip.mov). The zipping process is essential for the proper matching of cells along the anterior-posterior axis. As the two margins merge during dorsal closure the cells from each side of the epidermis that meet end up establishing permanent (adherens) junctions similar to those between the other epidermal cells. At the same time the actin corresponding to the cable segment they contained is de-polymerized and in the end there is no trace remaining of the fact that they were far apart at the outset of DC.

In this paper we propose a simple model for simulating DC. Our approach is a natural generalization of the one proposed in Hutson et al. (2003) which consists of a system of two Ordinary Differential Equations (ODE) obtained by considering the balance of the forces described above in a very symmetric setting where the LE is supposed to consist of the union of two arcs of circle. 
In that case it is sufficient to have one equation concerning the forces acting on the midpoint of each of these arcs plus a second equation to take the zipping into account. Here, we use a Partial Differential Equation (PDE) approach that allows us to go beyond these strong symmetry assumptions (which had already been relaxed in an ODE setting in Peralta, Toyama, Hutson, Montague, Venakides, Kiehart, and Edwards (2007)). A priori, the PDE setting allows us to consider quite general geometries and inhomogeneous forces. In practice, the full generality allowed by our approach can lead to optimizing a very big number of parameters which would be computationally too costly (and meaningless considering the precision of our present data). Thus, we will present the case where the forces are supposed to be determined by a small number of coefficients and by the geometry of the LE (which can be quite general), as described below. We have already applied this type of model to Drosophila embryo wound healing in Almeida, Bagnerini, Habbal, Noselli, and Serman (2009, http://hal.archives-ouvertes.fr/hal00480769/en/) obtaining quite satisfactory results (which confirms that this approach can deal with a wide range of hole geometries).

Each of the coefficients in our model is associated with one of the forces described and will thus give us a way of measuring the relative contribution of that force (they are our force parameters). In fact, we make a movie of each embryo going through DC and extract the contours corresponding to the position of the LE at different times. To take full advantage of the linearity of our model we start from the extracted contour at each time step and simulate its evolution during one time step to obtain a simulated contour which we compare to the extracted contour (experimental data) at the following time step. We will optimize the set of force parameters so that the simulated contours predicted by the model fit the best way possible (i.e. they minimize an appropriate cost function defined below) the real contours extracted from the film. This yields the force parameters of the embryo considered and is thus a way of measuring the relative intensities of these forces.

The way that these force coefficients change between native (sometimes also denoted as wild-type) and perturbed settings may give us a tool to identify which genes play an important role in regulating each of the forces present. Thus, we started by studying native (genotype arm-gal4, zipperGFP) embryos $(n=10)$ and checked that the set of parameters obtained for the different embryos was consistent. This is a strong indication of the pertinence of the model in this setting.

We further challenged our model to see what it yielded in a setting where 
the microtubule severing protein Spastin was ubiquitously expressed in the tissues using the armadillo-gal4 driver. Expression of this protein in engrailed half-segments of the lateral epidermis had been extensively studied in Jankovics and Brunner (2006) and they had observed that the zipping was significantly downregulated in this setting. In fact, as described in Jacinto, Wood, Balayo, Turmaine, Martinez-Arias, and Martin (2000), zipping requires the filopodia (and lamellipodia) whose formation depends on the actin organizing machinery which, in turn (as shown in Jankovics and Brunner (2006)), is linked to microtubule activity. Our study of Spastin embryos (genotype arm-gal4, zipper-GFP x UAS-spastin-GFP) $(\mathrm{n}=10)$ shows that the coefficient associated to zipping $\left(C_{4}\right.$ in our model $)$ we measured is considerably lower than that obtained in the native setting. This is consistent with the results of Jankovics and Brunner (2006) and comforts us in the choice of this model.

Another advantage of our model is the direct way it links the coefficients we measure with the intensity of the associated forces. For instance in the case of the decrease of the zipping coefficient in the Spastin setting that we measure, this means that the zipping force in the model is lower (since $C_{4}$ is the intensity of that force term) whereas a decrease in the zipping rate $k_{z}$ (as defined in Hutson et al. (2003)) just directly means that the shape is changing in a different way (and it is this that can then be indirectly associated with a difference in the zipping force).

The model we present here is supposed to be valid at a tissue level: it should be valid at correspondingly large space and time scales. This is why we don't describe the more complex phenomena implying cell rearrangement at the intersection of the segment boundaries with the LE (see Gettings, Serman, Rousset, Bagnerini, Almeida, and Noselli (2010)) nor the type of amnioserosa vibration behavior of Solon, Kaya-Copur, Colombelli, and Brunner (2009). It is clear that there are oscillations of the leading edge with small timescales (3 to 4 minutes) when compared to the 2 to 3 hours over which DC takes place. The type of model presented here is intended to describe the mean position of the leading edge at a larger timescale and not the fluctuations around this mean resulting from the vibrational behavior.

We thank one of the anonymous referees for bringing to our attention the existence of the recent paper Layton, Toyama, Yang, Edwards, Kiehart, and Venakides (2009) where a so-called second generation model for DC is proposed. The main motivation of that work is quite similar to that of the present one: to develop a PDE generalization of the ODE model of Hut- 
son et al. (2003) that is applicable in more general settings. However, our approaches are quite different and somewhat complementary. In fact, their work explores more the mechanical aspects with a very interesting comparison of the differences between having a linear or a hyperbolic relation between force and velocity. It also has a considerably larger number of parameters to describe mechanical properties of the tissues involved.

In Layton et al. (2009), the authors first recall the model developed in Hutson et al. (2003) and further extended in Peralta et al. (2007) for the case of asymmetric canthi to account for situations where the zipping occurs asymmetrically. Both references strongly lean on a constant leading edge velocity assumption, which happens to be violated during mechanical jump experiments (extensive laser ablation of the amnioserosa). Building upon the latter references, in Layton et al. (2009) force balance equations are adjoined to formulate a new model. Dropping the restrictive assumptions on the geometry of the leading edge (but staying within the restricted geometry of DC), the position of each of the margins is then made dependent on time and on the dorsal midline coordinate, allowing for more general amnioserosa traction and purse string contractile forces. The forces present are generated by biological force-producing elements, which consist of elastic, viscous and force-generating components but which are mostly positioned along the dorso-ventral direction with very little coupling along the antero-posterior direction. Canthi kinetics are also differentiated between posterior and anterior positions, and finally, the model parameters are identified through an optimization process like in our case, but with a cost functional that mostly takes into account the distance between the margins at the midpoint of the closure (in the spirit of the ODE approach in Hutson et al. (2003)) instead of the full geometry of the leading edge (as in the case of our PDE approach). Our approach has more naive mechanics but has more natural mathematics (the dorso-ventral direction is not so artificially singled out) with a truly multi-dimensional approach that can be easily extended to more general geometries like the ones one can come across in wound-healing (as studied in Almeida et al. (2009, http://hal.archives-ouvertes.fr/hal-00480769/en/)). In Layton et al. (2009) a comparison between simulations and experimental data for a native embryo is provided which show a remarkably good agreement. In the future, it will be interesting to combine our two approaches to put together their strong points and construct a more realistic model.

This paper is organized as follows: in section 2 we present and motivate our simple mathematical model; in section 3 we present the way we optimize 
the force parameters and the numerical methods used for computing the deformation fields and for simulating the movement of the LE; in section 4 we present the results we obtained for the force coefficients both in the native and the Spastin settings. In section 5 we apply the previous model Hutson et al. (2003) to our data sets in order to compare the results given by the two models and in section 6 we analyze the results obtained. Finally, materials and methods and the captions for the movies in the supplementary material are gathered in sections Appendix A and Appendix B.

\section{Simple Mathematical Model}

For mathematical purposes, the LE will also be denoted by $\omega$. Our simulation domain will be a rectangle, $M$, which contains the amnioserosa in the middle and part of the surrounding lateral epidermis all around it. The part of the domain occupied by the epidermis is $D=M \backslash W$ (see figure 1). The amnioserosa $W$ (and therefore also $D$ ) change in time. Actual mechanical behavior of the tissues involved should at least obey nonlinear visco-elastic dynamics, involving a chemo-bio-mechanical coupling, as introduced in Olsen, Sherratt, and Maini (1995), Olsen, Maini, and Sherratt (1998) and Murray (2003). However, as we mentioned above, we did not take into account short time (compared to the closure duration) dynamical behavior, like possible short period pulsing of the amnioserosa or vibrations of the LE (described in Solon et al. (2009)). Thus, one may neglect inertial forces and consider the overall process as a succession of linear elastic equilibria, where we omit as well the successive initial stress fields. We will adopt such a so-called quasi-static approach to describe this evolution.

At each step $i=1, \ldots, n$, let $W_{i}$ and $D_{i}$ denote, respectively, the positions of the amnioserosa and the ectoderm, and let $\omega_{i}=\omega_{i}^{t} \cup \omega_{i}^{b}$ that of the LE (see figure 1). We suppose (see below) the behavior of the epidermis to be linearly elastic, homogeneous, isotropic and subject to four forces:

1) the epidermal tension. It pulls the leading edges apart opposing the closure. We simulate it by constant normal forces applied at the top $\left(M^{t}\right)$ and bottom $\left(M^{b}\right)$ boundaries of our simulation domain. The actual forces should be considerably more complicated and are due to the stress fields induced by the previous morphogenetic movements (as well as those occurring during DC) but, for the moment, we use this rough approximation to have a simple description. 
2) The actin cable tension. This term gives rise to a force that is proportional to the curvature at each point and to the amplitude of the cable tension, in the spirit of what was described in Hutson et al. (2003) for the middle point of their arc of circle. In this paper we will suppose the cable tension $T$ to be constant (the general model is also valid with non constant cable tensions but, in that case, the tension variation will yield a tangential force along the cable proportional to the derivative of tension with respect to the arc-length). Here, we extend this force to all the points on the LE - we work in a PDE framework instead of the ODE framework used in Hutson et al. (2003). It points towards the exterior of $D_{i}$ (i.e. towards the interior of the amnioserosa $W_{i}$ ) at the points of positive curvature of the LE and towards the its interior at points of negative curvature.

3) A uniform normal force pointing towards the exterior of $D_{i}$. This force is associated to amnioserosa contraction (or lamellipodal crawling in a wound healing context - see Almeida et al. (2009, http://hal.archivesouvertes.fr/hal-00480769/en/)).

4) The zipping force at the canthi.

We will discuss a bit further this last term, i.e. the modeling of zipping, which plays a very important role in DC. As mentioned above (based on Jankovics and Brunner (2006)) this is the term that we expect to change significantly between the two settings we will consider in the present work (native and Spastin). In general, we can model the zipping force $(Z)$ by a term of the type

$$
Z(p)=\int_{\omega} f(p, q) \frac{q-p}{|q-p|} d q \text { for all } p \in \omega,
$$

where $\omega$ is the Leading Edge (LE), $p$ and $q$ are two-dimensional points belonging to $\omega$. Our present experimental tools and data do not enable us to obtain enough information about the function $f$ for making a more precise modeling of its contribution $(f(p, q)$ describes the intensity of the force with which the boundary points $p$ and $q$ are pulled towards each other by the filopodia). Nevertheless, it is natural to suppose that $f$ depends on the distance between the points $(|q-p|)$. Indeed, we can write, $f(p, q)=\phi(|q-p|) \psi(p, q)$, where the function $\phi: \mathbb{R}^{+} \rightarrow \mathbb{R}$ has compact support inside $|q-p|<2 L$, where 
$L$ is the maximum length of filopodia in the situation considered and the function $\psi$ has no special restrictions.

As mentioned in Almeida et al. (2009, http://hal.archives-ouvertes.fr/hal00480769/en/), this approach should also be appropriate for modeling the zipping in wound healing as long as the wound $W$ (the set enclosed by the LE in the wound-healing setting) is convex. In a general setting some caution is needed in defining which filopodia can interact.

In DC, not only we are often in a (nearly) convex setting, but we also have a particular geometry and behavior that simplifies the modeling of the zipping force. In the native setting (and most genetically modified settings we observed) there seems to be no zipping between points in the same margin, which implies that $f(p, q)=0$ if $p$ and $q$ belong to the same margin (so, in practice, the integration in (1) is only over the opposite margin). Moreover, the fact of filopodia interacting only with those coming from the opposite margin eliminates the problem that each margin is not convex near the canthi (the zipping zone).

There is even more structure that seems to constrain the zipping in DC: the epidermis of the embryo is divided into 14 segments separated by sharp boundaries, each of them constituted by anterior and posterior cells (green and red cells, respectively, in movie track.mov). This spatial organization is conserved at the end of DC by a proper segment adjustment potentially mediated by filopodia (Millard and Martin (2008)). Tracking of segment and parasegment boundaries during DC indicates that physical points on the leading edge move approximately vertically, i.e. orthogonally to the AP axis (figure 2).

If we want to take into account the fact that zipping plays a role in segment matching, then $f$ should also include specific information about the matching genes expressed by the cells present at points $p$ and $q$. In the native context, in the beginning of DC the parasegments that should match are placed (considering our orientation convention of horizontal AP axis and symmetry relative to this axis) vertically to their counterparts in the opposite leading edge. Moreover, as shown in figure 2, our experiments indicate that they move approximatively vertically throughout DC. In this work, we will consider only cases where we have this property, and simulate them by supposing that we have a vertical zipping force which is supported in the part of the leading edge where the vertical distance between the two margins is smaller than $2 L$. To be precise, for each time point $i$, we suppose that the upper leading edge (denoted $\omega_{i}^{t}$ ) is described by a function $y=g_{i}(x)$ 


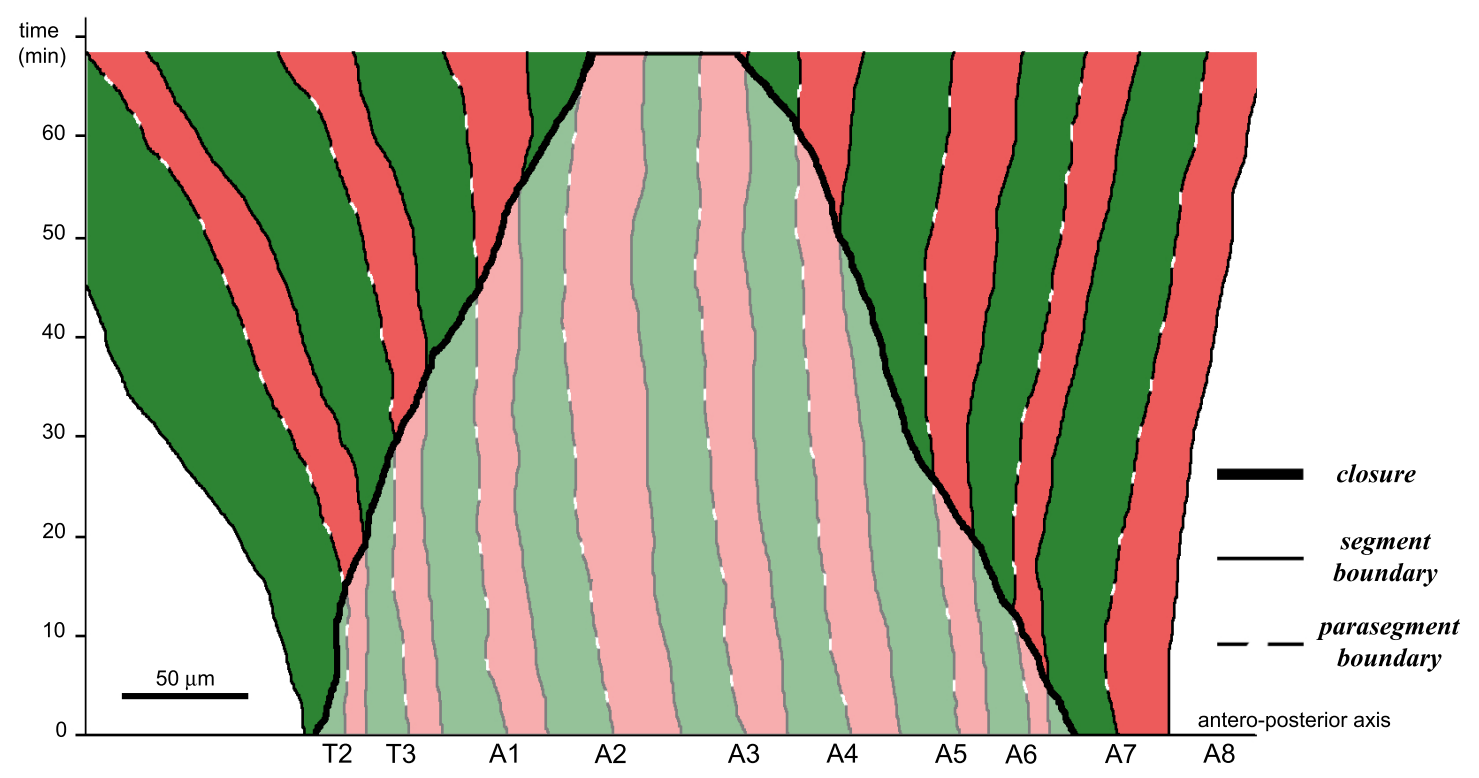

Figure 2: Antero-posterior stretching of segmental boundaries during dorsal closure. The central curve (bold line) shows the closure of each parasegmental and segmental boundaries during time, from a total opening (the exposed amnioserosa extends from segment T2 to segment A7 at time 0) nearly up to the end of closure (the exposed amnioserosa covers only parts of segments A2 and A3 at time 70'). The solid and dashed lines indicate the positions of segmental and parasegmental boundaries at each time, respectively. These curves were obtained by tracking the half-segment boundaries during the dorsal closure of the embryo shown in movie track.mov. 
and the lower leading edge (denoted $\omega_{i}^{b}$ ) by $y=h_{i}(x)$ for $x \in\left[c_{a}, c_{p}\right]$ where $c_{a}$ and $c_{p}$ are the $x$ coordinates (horizontal positions) of the anterior and posterior canthi at time point $i$. The zipping will then be effective only in the set $Z_{i}:=\left\{(x, y) \in \omega_{i}: g_{i}(x)-h_{i}(x) \leq 2 L\right\}$.

Treating the case where the zipping is stronger at one canthus than at the other (as was done in an ODE setting in Peralta et al. (2007)) is also easy in this model - it suffices to introduce one zipping parameter for the anterior canthus and another one for the posterior canthus. However, for both native closure and that of Spastin embryos considered in this paper, one single zipping parameter is sufficient for obtaining a good agreement. We will consider more complex situations in the future.

Coming back to the global model of DC, the cytoskeleton and membrane of the cells of the epidermis are globally viewed as a mechanical continuum, which can bear traction and compression loads but not bending nor torsion. Assuming a linear elastic response of this medium considered as a plane surface, the elastic deformations are then in-plane. The model derived from elastic thin shell theory results in a coupled system, formed of a Laplace term and a coupling term between the two planar displacements. To keep our model simple, we rather use a so-called membrane model (uncoupled, keeping only the Laplace operator) governed by a Poisson equation with suitable boundary conditions (in particular, applied forces are introduced as Neumann boundary conditions). This type of model is widely used in the shape identification framework (see Allaire (2007)). Our approach, though being mechanically questionable, turned out to be well suited from a computational viewpoint, allowing us to perform an efficient parameter identification task, and yielding a good predictor model.

We adopt the convention that the first image we consider in each film is time point 1 for that film so that time point $i$ will just be image number $i$ being considered. The simulation time step $i$ will be the one starting at time point $i$ and yielding a simulated contour at time point $i+1$.

Therefore, in the simple model implemented in this paper, we assume that at each time step $i$, the corresponding displacement field $u_{i}$ will satisfy (see figure 3) 


$$
\left\{\begin{array}{l}
-\Delta u_{i}=0 \text { in } D_{i}, \\
u_{i}=0 \text { on } M^{l} \cup M^{r}, \\
\frac{\partial u_{i}}{\partial n}=C_{1} n \text { on } M^{t} \cup M^{b}, \\
\frac{\partial u_{i}}{\partial n}=C_{2} \kappa n+C_{3} n \text { on } \omega_{i} \backslash Z_{i}, \\
\frac{\partial u_{i}}{\partial n}=C_{3} n+\left(\begin{array}{c}
0 \\
-C_{4}
\end{array}\right) \text { on } \omega_{i}^{t} \cap Z_{i}, \\
\frac{\partial u_{i}}{\partial n}=C_{3} n+\left(\begin{array}{c}
0 \\
C_{4}
\end{array}\right) \text { on } \omega_{i}^{b} \cap Z_{i} .
\end{array}\right.
$$

where $n$ is the external unit normal vector to $\partial D_{i}, \kappa$ is the curvature of $\omega_{i}$, $Z_{i}$ is the effective zipping zone defined above and $C_{1}, C_{2}, C_{3}, C_{4}$ are constant parameters (in this simple setting) which are determined using the experimental data. The term $C_{1} n$ represents the ectoderm resistance force density on the top $\left(M^{t}\right)$ and bottom $\left(M^{b}\right)$ edges of our simulation rectangular domain (the exterior normal, $n$, points vertically upwards on the top edge and vertically downwards on the bottom one - notice that this corresponds to the dorsoventral direction of the original fly embryo); $C_{2}$ corresponds to the cable tension (the effective force density acting on each point of the cable being given by $C_{2} \kappa n$, where $\kappa$ is the curvature of the LE at the point and $n$ is again the exterior normal to $D_{i}$, now along it's inner boundary - which is thus pointing into the amnioserosa); $C_{3} n$ represents the force density along the LE due to amnioserosa contraction, where $n$ is as in the previous case; finally, $C_{4}$ is the amplitude of the force density due to zipping (this term is active only on the zipping zone near each of the canthi).

Since we do not know the Young modulus of the tissues involved, the coefficients $C_{1}, \ldots, C_{4}$ can only be determined up to this unknown value (which is taken as unit-valued and thus not even appearing in equation 2 to make things simpler). Therefore, in this work, these coefficients are presented as numerical values (without physical units). This is sufficient for our analysis since it is the relative variation of these coefficients between the native and the modified setting that is used to detect phenotypes. 


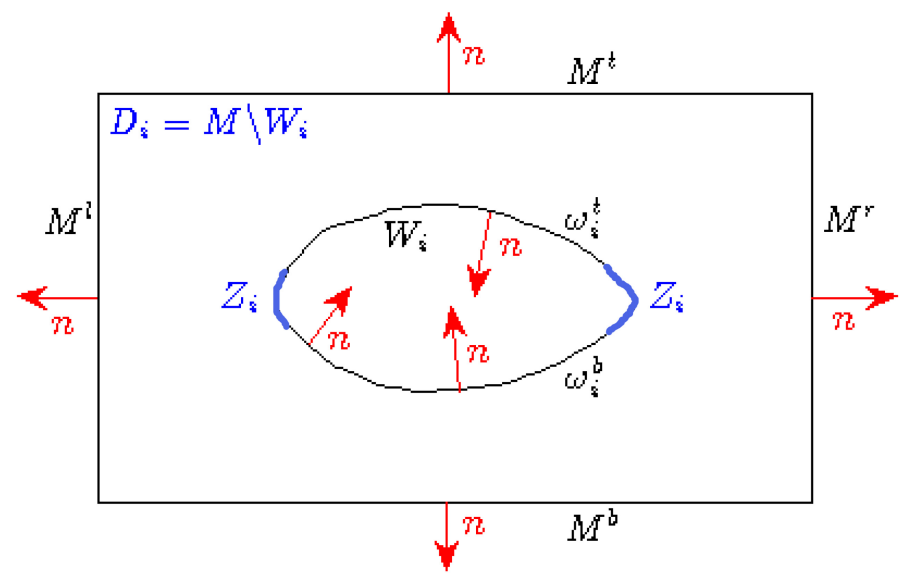

Figure 3: The simulation domain $D_{i}$, the two components of the zipping domain $Z_{i}$ (in blue) and the exterior normal vector field $n$ on its boundary (in red).

We notice that allowing for a more general operator (instead of the Laplacian) and for time and space dependence of the coefficients gives us the possibility of going beyond the linear elastic, homogeneous and isotropic setting that, for simplicity, we will consider in this paper.

As we mentioned, in our model forces appear as Neumann boundary conditions since they should be equal to the normal component of the stress tensor field, $\sigma_{n}=\sigma \cdot n$ which, with our assumptions, in time step $i$ reduces to $\nabla u_{i} \cdot n=\frac{\partial u_{i}}{\partial n}$.

Once $u_{i}$ is obtained, we consider its restriction the leading edge (LE) $\omega_{i}$ and displace this boundary (using a level set method) to obtain $\omega_{i+1}$. The domain $W_{i+1}$ enclosed by $\omega_{i+1}$ is the new position of the LE. More precisely, we compute the solution $u_{i}$ of $(2)$ in the domain $D_{i}$ by using finite element methods on a triangular mesh. We obtain a vector field $u_{i}$, which is used as a velocity vector in the level set method in order to move $\omega_{i}$ (see figure 4 ) and obtain $\omega_{i+1}$.

The position of $\omega_{i+1}$ obtained also defines the new epidermal domain $D_{i+1}=M \backslash W_{i+1}$ which could be used in the following step to solve equation (2) in order to obtain $u_{i+1}$, and so on. This would be the most natural way of implementing our model in a general setting. However, as we said in the introduction and will explain better in the following section, in this paper we use $W_{i+1}$ to compare with the experimental contour at time point $i+1$ and evaluate the error of the simulation time step but, to take full advantage 

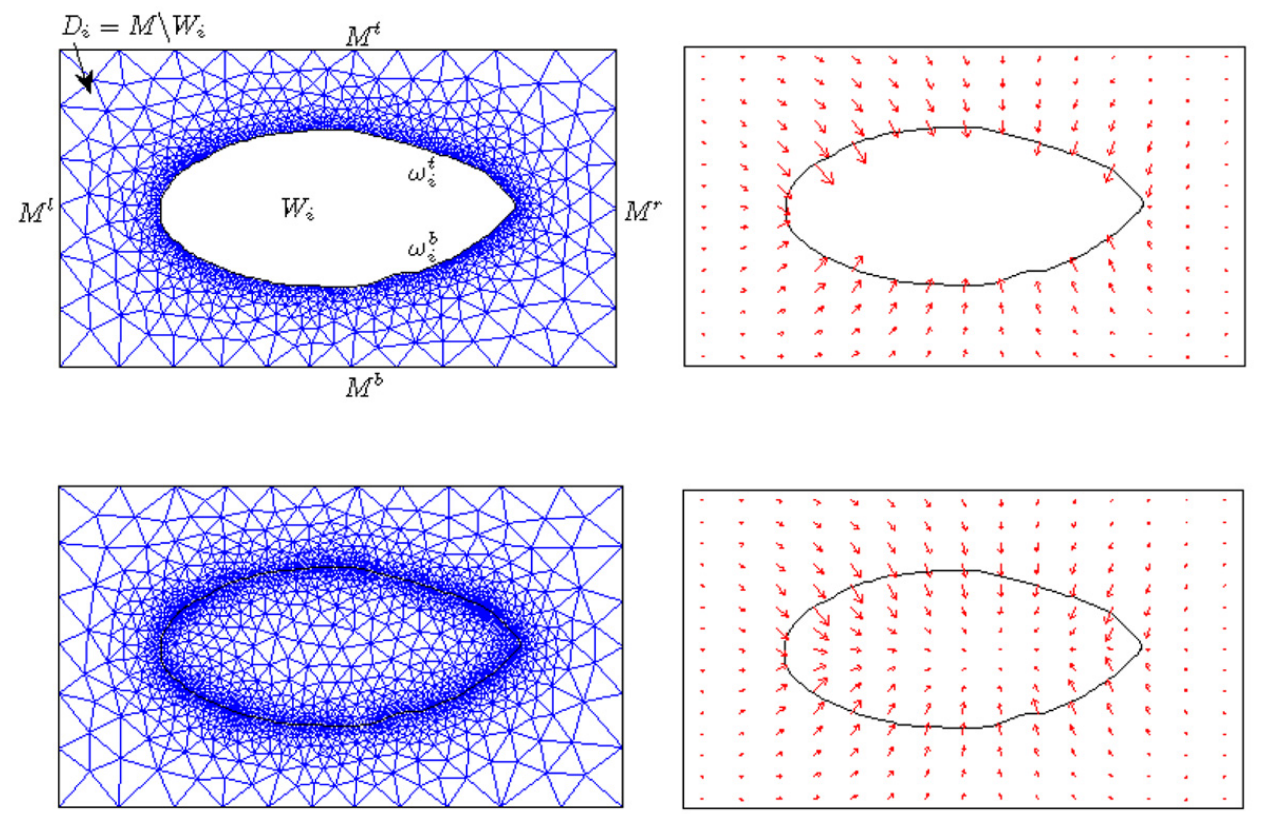

Figure 4: Example of a computed solution $u_{i}$ alone (top right) and together with its associated extension $u_{i}^{\text {int }}$ (bottom right) and the corresponding computational meshes (left). 
of the linearity of the model, we will take the experimental contour at time point $i$ to initiate the simulation time step $i$.

We remark that we are doing all our analysis in 2D while the real dynamics is 3D. In fact, we try to obtain our original data keeping as much 3D information as possible (although the embryos are still flattened by their own weight - see Appendix A) and avoiding to interfere with the dynamics (in preceding works the embryos were squeezed between the coverslip and a permeable membrane which, although it has the advantage of reducing the spatial shifts, risks perturbing the movement and destroys the $3 \mathrm{D}$ character). However, for the moment, we project the confocal images to obtain 2D data which is simpler to treat (in particular for the contour extraction). Nevertheless, all the forces described in the model can be considered in 3D (in that case, at each point, one should naturally work on the tangent plane to the embryo at the point considered and project all the forces onto that plane).

The model presented here is a macroscopic model that does not take into account the positions of the individual cells constituting each of the tissues considered. Thus, we essentially expect to be able to use it to obtain results at a tissue level scale and not at a cellular scale where the geometry of each cell and its neighbors should also play an important role. However, recent and ongoing work shows that medial (bulk) actin and myosin (see, for instance, Martin, Kaschube, and Wieschaus (2009) and Ma, Lynch, Scully, and Hutson (2009)) play an important role in the mechanical behavior of the tissue (contradicting previous approaches that supposed that it was mainly due to the junctional acto-myosin network which concentrates along each cell's boundaries with its neighbors). The scale of the bulk actin network is considerably smaller than individual cell size - this should be one of the main reasons why continuous approaches, like the one presented here, yield good results at a considerably smaller scale than what one would expect if the mechanics was coming mainly from cell edges. Moreover, even for more precise cellular level studies where we have to concentrate on a small patch of cells, not being able to follow in detail all the cells in the tissue considered, this type of macroscopic models can be useful for providing reasonable boundary or asymptotic conditions for the local problems.

In the same spirit, this model assumes a very simple mechanical behavior of the tissue. Our original motivation for choosing a homogeneous, isotropic and linear elastic model for the epidermis was, on the one hand, the fact that the precision of our present data did not justify more complicated assumptions and, on the other hand, the simplicity of the equations obtained 
(and, thanks to the linear aspect of the model, the great reduction that this yields for the parameter optimization procedure - see section 3). However, very recently, new techniques have been developed, which may make it possible to obtain more precise information about the forces involved. As a matter of fact, the measures concerning relaxed strain patterns and radial displacement (for hole drilling experiments in amnioserosa cells during DC) published in Ma et al. (2009) comfort us in our choice of this simple model at a macroscopic scale since they indicate that:

- correlation with the individual geometry of the cell walls is significant only for distances below first neighbor distance (10 $\mu \mathrm{m}$ in their case), and quickly becomes considerably more isotropic beyond this distance;

- a linear elastic, isotropic and homogeneous sheet approach gives a good prediction of the observed radial displacements for distances above 10 $\mu m$.

Nevertheless, when the mechanical and 3D properties of the tissue can be determined in a more precise way, it will be interesting to include them to improve the present model.

\section{Numerical Simulations}

As said in the previous section, we assume that the dynamics of the Leading Edge is governed by equations (2) where the magnitude of the parameters $\left(C_{j}\right)_{1 \leq j \leq 4}$ have to be identified. Let $C=\left(C_{1}, C_{2}, C_{3}, C_{4}\right)$. The aim of the numerical simulations is to identify such global coefficients $C$ by solving an unconstrained optimization problem in both a native and a modified setting, in order to validate our model.

First, we obtain experimental successive positions of the Leading Edge by means of image processing/contour-extraction techniques. We consider at each time step $i$ the experimentally observed position of the LE, $\omega_{i}^{E X P}$, as initial position. For a given collection of parameters $C$, we solve equation (2) in order to obtain a displacement $u_{i}$, which is used to evolve the initial position $\omega_{i}^{E X P}$ of the LE into an updated one $\omega_{i+1}^{P D E}$. Let $W_{i}^{E X P}$ and $W_{i}^{P D E}$ denote the sets enclosed by $\omega_{i}^{E X P}$ and $\omega_{i}^{P D E}$, respectively.

Since in the simple application of this model which will be implemented in this paper we want to identify constant (space and time independent) force 
coefficients $C$ which describe the whole process of closure, we consider the optimization problem consisting of minimizing the cost function $J$ given by

$$
J(C)=\sum_{i=1}^{m}\left|W_{i+1}^{E X P} \Delta W_{i+1}^{P D E}\right|,
$$

where the expression $\left|W_{i}^{E X P} \Delta W_{i}^{P D E}\right|$ denotes the area of the symmetric difference set between $W_{i}^{E X P}$ and $W_{i}^{P D E}$, i.e. the area of set of elements which are in one of these sets, but not in both (exclusive disjunction in Boolean logic). The symmetric difference provides a quantification of the error to be minimized in order to obtain the correct values of the parameters $C$. Here. $m$ is the number of time evolution steps considered in the optimization procedure and the cost of each step is evaluated at its end which explains why we are evaluating the symmetric difference at time point $i+1$ to obtain the cost of the error made in step $i$.

We use a global optimization approach, based on the Genetic algorithm and Direct Search (GADS) toolbox (ga) provided by Matlab, to obtain a solution. We eventually refine this minimizer by performing a direct search optimization (patternsearch) around the previously found solution.

The genetic algorithm allows to solve both constrained and unconstrained optimization problems by mimicking biological evolution. Roughly speaking, at each step, the algorithm randomly selects individuals in a population and uses them, through cross-over, mutation and best fit selection, to generate the children for the next generation. In this way the population evolves toward an optimal solution. It is a suitable method for problems where standard optimization algorithms fail to converge since the objective function is for instance discontinuous, non differentiable or highly nonlinear. In our case, the cost function $J$ is obtained by solving a PDE and then computing the area of the symmetric difference between the approximated contour and the experimental one. Therefore, $J$ is non-smooth and classical gradient methods are useless. Moreover, a priori one cannot exclude the existence of multiple local minima, for which descent methods are inefficient. The genetic methods overcome these two drawbacks, but it is necessary to carefully choose the parameters (e.g. the population size, the amount of mutation, the crossover rate, etc.) to obtain accurate results. For instance, one crucial factor is the diversity of the population: if the diversity is too high or too low, the genetic algorithm might not perform well.

A well known shortcoming of the above global minimizing algorithms is 
that they use extensive call to cost evaluation, which in our case amounts theoretically to solve, for each time step $i$, a big number of large scale linear systems coming from the finite element discretization (as many as the number of generations times the number of individuals per generation).

Fortunately, we can take advantage of the linearity of (2) to solve just one partial differential equation for each significant parameter: for $j=1, \ldots, 4$ and for each time step $i=1, \ldots, m$, we define $u_{i}^{j, 1}$ to be the solution of problem (2) with $C_{j}=1$ and $C_{k}=0$ for $k \neq j$. Then, thanks to the linearity of our model, the solution of the full problem (2) will be given by

$$
u_{i}=\sum_{j=1}^{4} C_{j} u_{i}^{j, 1}
$$

To take full advantage of this linearity, in this paper where we are assuming the coefficients $C_{j}$ to be constant (in order to have a small number of parameters), we will always take as $D_{i}$ the experimental contour at time point $i$ (i.e. the one obtained using as inner boundary $\omega_{i}$ the contour extracted from the image number $i$ ) of the film we are treating. This way, the domain itself is also independent of the values of the coefficients $C_{j}$ being used, and thus the same basis of solutions is valid at step $i$ independently of the different evolutions that are obtained for different choices of the coefficients $C_{j}$ in the previous steps. We then use the model to make the contour evolve during one time step and obtain a simulated contour at time point $i+1$ which we compare with the experimental contour at that time point.

We remark that even without starting from the experimental contour at each time step, the linearity of the model still reduces significantly the number of PDE to solve in the case where the coefficients $C_{j}$ can change in time.

Once we have the basis of solutions $u_{i}^{j, 1}$, for $j=1, \ldots, 4$, the general solution $u_{i}$ will just depend linearly on the parameters $C_{j}$ which simplifies considerably their optimization.

Moreover, as we will see in the following section, we will in practice be including the effect of the amnioserosa contraction, which in our model should be measured by $C_{3}$, in the coefficient $C_{2}$ originally corresponding to the actin cable tension (since the two effects are hard to distinguish in this geometry). Therefore, we will take $C_{3}=0$ and we just have to compute $u_{i}^{1,1}, u_{i}^{2,1}$ and $u_{i}^{4,1}$. 
The numerical simulations are performed in Matlab code (http://www.mathworks.com/) and by using Comsol Multiphysics software (http://www.comsol.com/) to compute the numerical solution of (2) and (7) by the finite element method.

The finite element method (see for instance Quarteroni (2009)) is the most used numerical technique to approximate solutions of partial differential equations. The computation domain is subdivided into smaller regions, typically triangles or quadrilaterals in two dimensions. Over each element of the mesh, the unknown variables of the PDE are computed using polynomial expansions which depend on the nodes used to define the finite element shape. Instead of directly discretizing the PDE, finite element method consists in multiplying the equation by a test function, chosen in a appropriate function space, integrating over the computation domain and then using the integration by parts to transfer the derivatives onto the test function. In this way, the boundary value problem is transformed into an equivalent form, called weak or variational form, which requires less regularity. The weak form is then discretized by choosing a subspace of piecewise polynomial functions (generally quadratic) which leads to solving a set of linear equations. In our case, we use a triangular mesh, quadratic polynomial functions and Umfpack direct solver for computing the solution of the linear system.

Having obtained $u_{i}$, we use level set methods to perform the evolution of our contour (Osher and Sethian (1988)). Level set methods (also called dynamic implicit surfaces) (Sethian (1999), Osher and Fedkiw (2003), Kimmel (2004)) are a set of popular numerical algorithms for solving a particular class of first-order hyperbolic partial differential equations, called HamiltonJacobi equations (HJ). They are used for tracking and simulating the motion of dynamic surfaces (in two and three dimensions) in many fields as image processing, computational fluid dynamics, seismic analysis and material science. In this framework, the interface front (which, in our case, represents the position of the LE) is implicitly represented as the zero level set of a function $\Phi: \mathbb{R}^{2} \times \mathbb{R}^{+} \rightarrow \mathbb{R}$ (or $\Phi: \mathbb{R}^{3} \times \mathbb{R}^{+} \rightarrow \mathbb{R}$ in three dimensions), i.e. the front is given by $\Phi(x, t)=0$ (see figure 5 ). If $x(t)$ represents the path of a point on the propagating front, differentiating with respect to $t$, we obtain the Hamilton-Jacobi equation

$$
\partial_{t} \Phi(x, t)+\dot{x} \cdot \nabla \Phi(x, t)=0
$$

where $\dot{x}:=\frac{d x(t)}{d t}$ (the Lagrangian material particle velocity). 

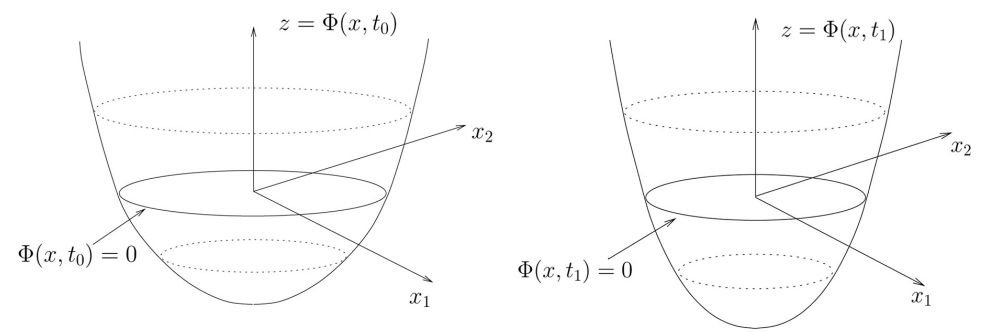

Figure 5: At each time $t$ the propagating front is the zero level set of a function $\Phi(x, t)$ solution of a Hamilton-Jacobi equation (5).

Level set methods consist in solving (5) and then computing at each time step the propagating front as zero level set of $\Phi$. This approach requires an initial function $\Phi(x, t=0)$ with the property that the zero level of this initial function corresponds to the initial position of the front: a possible simple choice is given by the signed distance to the front (negative inside the set enclosed by the front and positive outside this set).

There are three main advantages in evolving interfaces by level set methods instead of implementing particle or Lagrangian methods: changes of topology are naturally handled and surfaces automatically merge and separate; geometric quantities, like the surface normal, the curvature, etc. are easy to compute; the extension to three and even higher dimensions is conceptually straightforward.

In our particular case, at time step $i$, we apply level set methods to displace the front (representing the position of the LE) from its initial position $\omega_{i}$ using the displacement field (obtained by solving equation 2). Thus, for each point in $x \in \omega_{i}$ the material particle velocity should be $u_{i}(x)$, and the function $\Phi_{i}(x, t)$ we want solves

$$
\partial_{t} \Phi_{i}(x, t)+u_{i}(x) \cdot \nabla \Phi_{i}(x, t)=0 .
$$

We remark that in this equation $t$ is just an artificial parameter for the level set flow and does not represent real time. In fact, the only precaution is to always follow the flow up to the same $t$ value (which, for this paper, we chose to be $t=0.1$ ) so that we can compare the coefficients obtained for the different embryos considered (in this paper, all the native and all the Spastine ones treated).

The implicit representation of $\Phi_{i}$ displaces all the level sets throughout the entire computational domain, not only the zero level set. Hence, we must 
be able to construct an extension velocity which, starting with the velocity prescribed at the interface, builds an appropriate velocity field everywhere in the computational domain. This extension is in general not straightforward and different approaches are proposed in the literature (see Sethian (1999)). The main requirement for an extension velocity is that it smoothly approaches the prescribed interface velocity near the zero level set. In our case the velocity field, which is already defined in $D_{i}$ as the solution of 2 , has a natural extension in the domain inside the inner boundary $\omega_{i}$ (i.e. in the epithelial hole filled by the Amnioserosa $\left.W_{i}\right)$ : the harmonic extension obtained solving

$$
\left\{\begin{array}{l}
-\Delta u_{i}^{i n t}=0 \text { in } W_{i} \\
u_{i}^{i n t}=u_{i} \text { on } \omega_{i}
\end{array}\right.
$$

In this way, we obtain an extension of the original vector field $u_{i}$ to the entire rectangular domain (which we will still denote by $u_{i}$ for simplicity). We then solve the HJ equation (6) on a regular cartesian grid by using a second order numerical finite differences scheme both in space and in time. The value of $u_{i}$ in the regular grid is computed by interpolating $u_{i}$ on a triangular mesh. Due to the hyperbolic character of equation (6), upwinded approximations or artificial viscosity must be used in order to maintain stability. If the upwind propagation direction can be computed, upwind schemes yield more accurate results than artificial diffusion. At each grid point, the upwind method corresponds to approximating $\partial_{x} \Phi_{i}$ and $\partial_{y} \Phi_{i}$, the $x$ and the $y$ components of the gradient of $\Phi_{i}$, by left or right finite differences according to the direction of propagation, i.e. to the sign of the $\mathrm{x}$ and the $\mathrm{y}$ component of $u_{i}$. For instance for the x-component, the left $D^{-} \Phi\left(x_{j}\right)$ and right $D^{+} \Phi\left(x_{j}\right)$ first-order accurate approximation at node $\left(x_{j}, y_{k}\right)$ are

$$
\begin{aligned}
D^{-} \Phi\left(x_{j}, y_{k}\right) & =\frac{\Phi\left(x_{j}, y_{k}\right)-\Phi\left(x_{j-1}, y_{k}\right)}{\Delta x} \\
D^{+} \Phi\left(x_{j}, y_{k}\right) & =\frac{\Phi\left(x_{j+1}, y_{k}\right)-\Phi\left(x_{j}, y_{k}\right)}{\Delta x}
\end{aligned}
$$

We perform spatial discretization by using an upwind second order Essentially Non-Oscillatory (ENO) scheme (Osher and Shu (1991), (Kimmel, 2004, chap. 3)) which consists of making the second order left and right correction to the first order approximation (8) and then choosing the least oscillatory of the two. The time discretization is treated by a second order total variation diminishing Runge-Kutta scheme. We use the Matlab tool- 
box of Ian M. Mitchell (Mitchell (2008)) (http://www.cs.ubc.ca/ mitchell) to implement level set methods.

\section{Experimental results}

In order to validate our model we applied it in a native and also in a disrupted setting (Spastin embryos) which had already been described in the literature (Jankovics and Brunner (2006)). We used ten embryos of each type and filmed them during dorsal closure. The LE positions were extracted from these films yielding the experimental contours (the $\omega_{i}^{E X P}$ of the previous section). The coefficients $C_{j}, j=1,2,4$ for each embryo were then obtained using our model, as described above (see figure 6). We recall their meaning

$C_{1}$ is associated with ectoderm resistance;

$C_{2}$ is originally associated to actin cable tension but which will also include the effect of amnioserosa contraction since in our analysis we are dropping $C_{3}$;

$C_{4}$ corresponds to zipping force intensity.

As we mentioned before, we chose to drop the $C_{3}$ term (the one associated with amnioserosa contraction in our original model) since in the Dorsal Closure settings studied here the second and third forces considered (curvature force corresponding to cable tension and constant normal force associated to amnioserosa contraction) are collinear and have nearly constant relative magnitudes (since, in the sweeping phase of DC and in the native and the Spastin setting considered here, the curvature has small variations in the central part of the LE, far from the canthi). As these two terms play nearly interchangeable roles in the settings considered, we have an approximately invariant direction of the cost function which makes it meaningless to optimize $C_{2}$ and $C_{3}$ simultaneously. This will no longer be the case when we have more complicated geometries that appear in genetically modified settings that strongly affect DC (or in wound healing).

We gathered in figure 6 the results obtained using our method as described above for the optimized coefficients $C_{1}, C_{2}, C_{4}$ of ten native and ten spastin embryos. In figure 7 we show the averages and standard deviations of each of the data sets presented. We will analyze this results in section 6 but notice right away that, as expected, we obtained a significant downregulation of the zipping coefficient in the Spastin setting. 

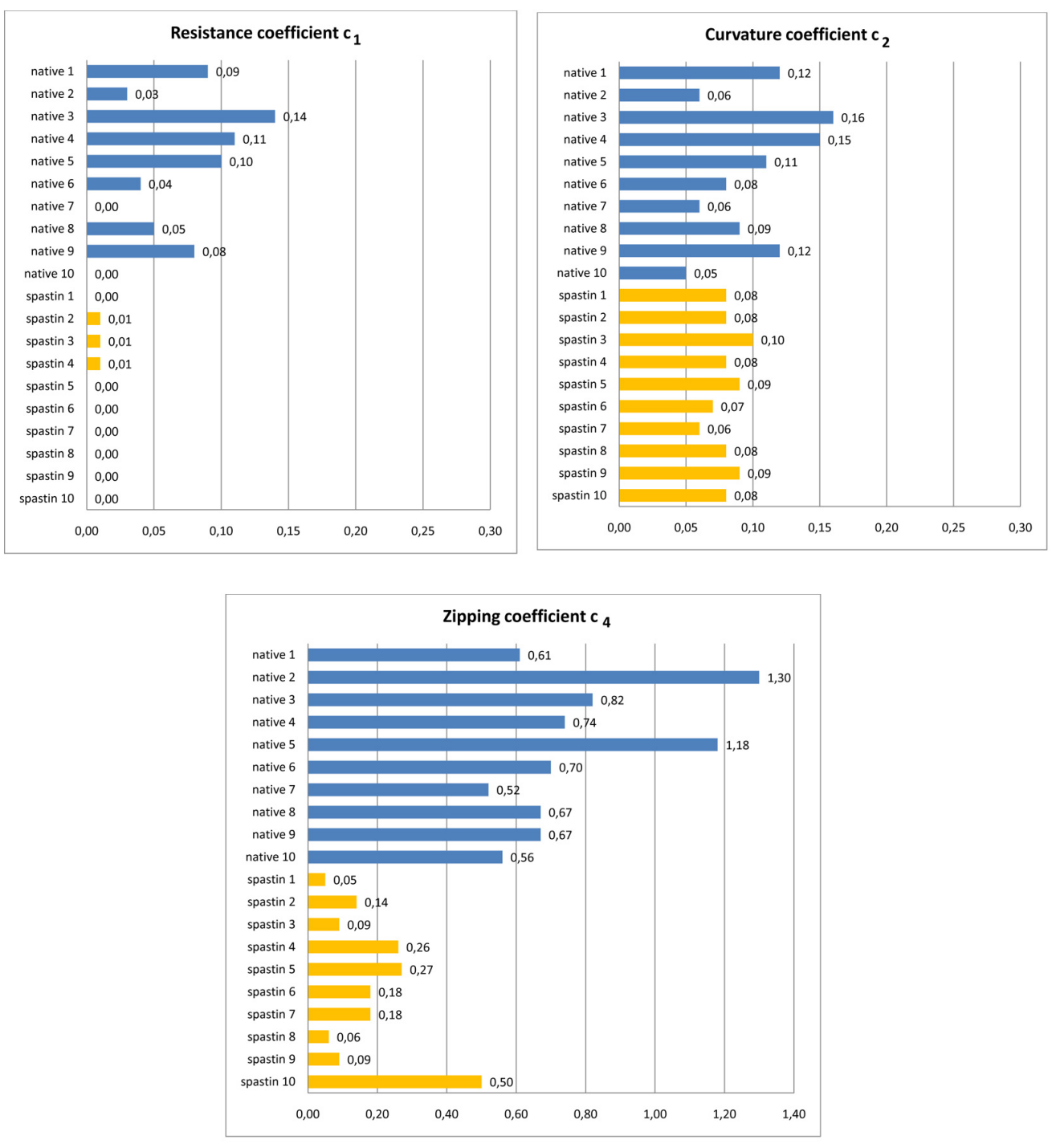

Figure 6: The optimized coefficients $C_{1}, C_{2}, C_{4}$ of ten native and ten modified (arm-gal4 x UAS-spastin-GFP) embryos. 

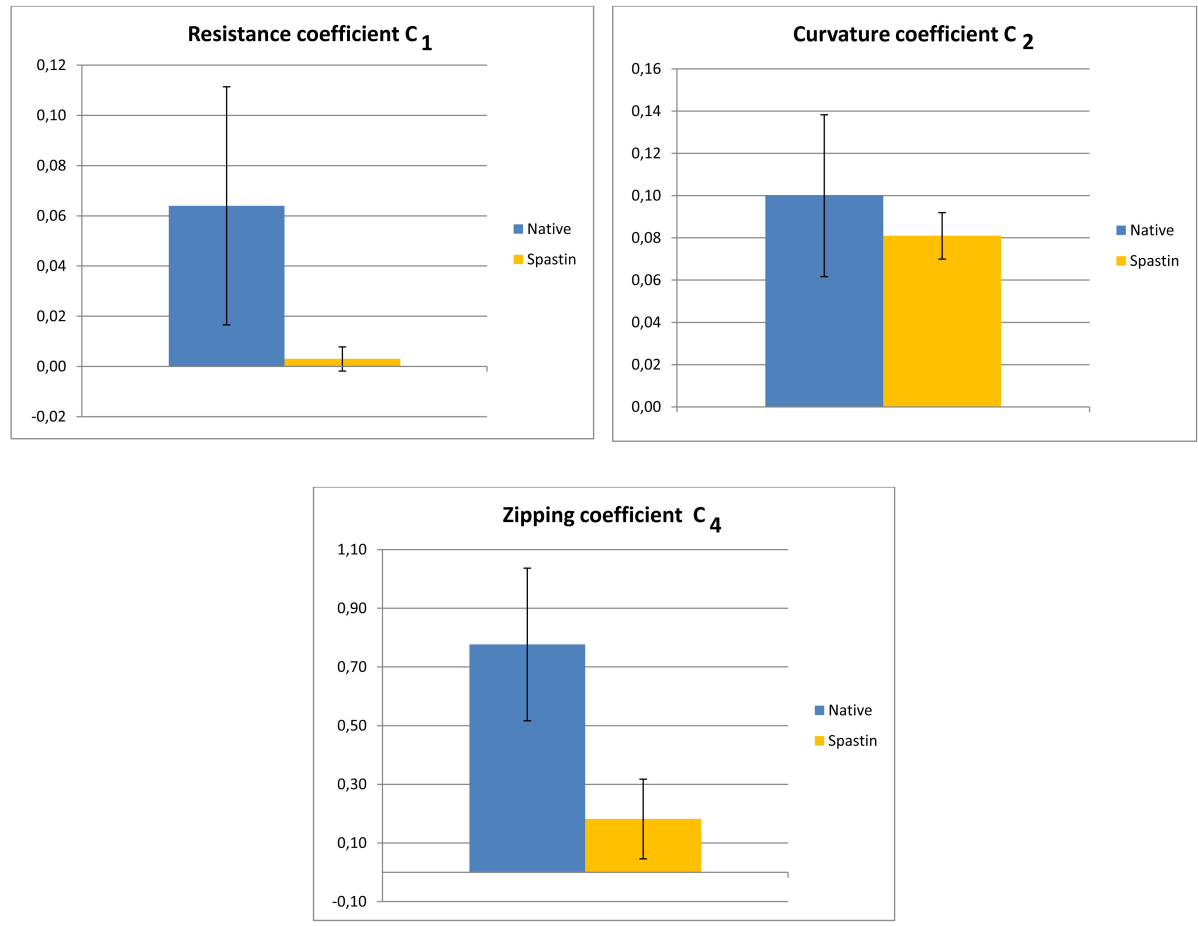

Figure 7: Average of the optimized coefficients $C_{1}, C_{2}, C_{4}$ of ten native and ten modified (arm-gal4 x UAS-spastin-GFP) embryos. Error bars represent the standard deviation of the data in each class. 

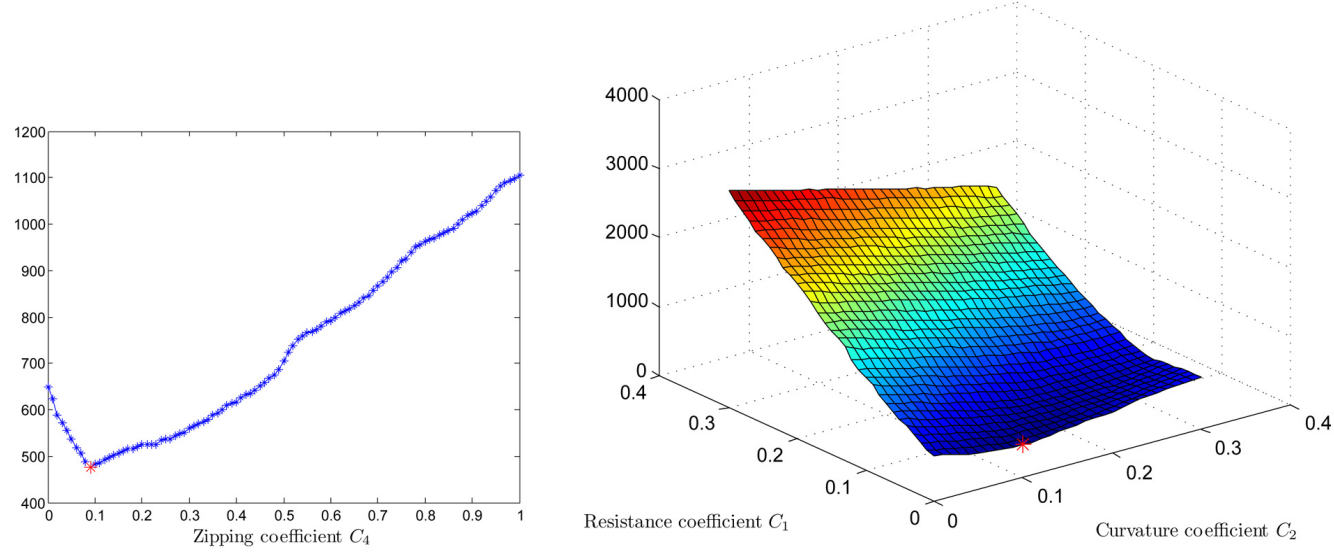

Figure 8: On the left, for each fixed value $C_{4}$, we show for Spastin embryo 3 the minimum of the two dimensional function $J\left(\cdot, \cdot, C_{4}\right)$ in the interval $[0,0.3] \times[0,0.3]$, computed with constant step size 0.01 . On the right, we show the cost surface $J(\cdot, \cdot, 0.09)$, i.e. corresponding to the minimum $C_{4}=0.09$ (the red point $\left(C_{1}, C_{2}\right)=$ $(0.01,0.1)$ minimizes the section $J(\cdot, \cdot, 0.09)$ of the cost function for this embryo).

We also studied the stability of our model by computing the cost function $J$ for an adequate number of values, in order to check the continuous dependence of $J$ on the parameters and check that we do not have a great number of local minima where the minimization procedure could get trapped. In particular, for Spastin embryo 3 we computed $J\left(C_{1}, C_{2}, C_{4}\right)$ with $C_{1}$ and $C_{2}$ varying from 0 to 0.3 and $C_{4}$ varying from 0 to 1 with constant step size 0.01. A partial representation of these values is shown in figure 8 . This figure gives a clear indication that, at least in this case, it is unlikely for $J$ to have significant oscillations or multiple minima and that it depends continuously on the parameters. In particular, it is quite reassuring to see that the profile of minimum of the cost function $J\left(\cdot, \cdot, C_{4}\right)$ (which is a function of $C_{4}$ alone represented in the left of figure 8) around the value of $C_{4}$ the minimizes it (which will be the one chosen by our model) is not flat at all. We notice that this type of computation for obtaining the corresponding surface would not have been reasonably feasible if we were using a nonlinear model. For this embryo, the coefficients obtained by the genetic algorithm are $C=\left(C_{1}, C_{2}, C_{4}\right)=(0,0.06,0.18)$ (shown in figure 6$)$. 


\section{Comparison with the previous model Hutson et al. (2003)}

In the previous model Hutson et al. (2003), the margins were approximated by two arcs of circle and the evolution was described by a system of two ODE for the height $H(t)$ (maximum dorso-ventral distance between the two margins of the leading edge) and the width $W(t)$ (antero-posterior distance between the two canthi) of the dorsal opening. It has explicit solutions given by

$$
\begin{gathered}
H(t)=H(0)-V t \\
W(t)=W(0)\left[1-\frac{V t}{H(0)}\right]^{\frac{k_{z}}{2 V}} .
\end{gathered}
$$

where $H(0)$ and $W(0)$ are the initial height and width, and $V$ and $k_{z}$ are two parameters. The first one, $V$, represents the vertical velocity, i.e. the rate of change of the height $H(t)$, which is quite well approximated by a constant in the native setting (in the Spastin setting the fit is slightly worse, but still acceptable). The second, $k_{z}$, is the zipping coefficient associated with the way the filopodial zipping acts near the canthi.

We applied this model to our data set, to compare with the results given by our own model. For each embryo considered we first measured, for each time, the height $H(t)$ and the width $W(t)$ of the dorsal opening. Next, we did a linear regression to find the slope $-V$ of the straight line that fitted best (minimum square deviation) the experimental points for $H$ as a function of time (in the spirit of equation 9). Finally, using the $V$ found in the previous step and the values of $V(0)$ and $H(0)$ measured in the first frame of the film, we did a nonlinear regression (again minimizing mean square deviation) to obtain the exponent $k_{z}$ that gives the best fit in the exponential law of equation 10. We used Matlab to perform linear and nonlinear regression, but there are many equally good choices.

In this way we obtained the two parameters, the vertical velocity $V$ and the zipping coefficient $k_{z}$ that describe DC in the model of Hutson et al. (2003). We present these results in figure 9. One can see that both the height and the zipping coefficients tend to be slightly smaller for Spastin

embryos than in native closure, but that the data corresponding to the two settings have some overlap.

For comparison with the results of our model shown in figure 7, we also present the corresponding averages obtained using the model of Hutson et al. (2003) in figure 10. We can see that both the average vertical velocity and 

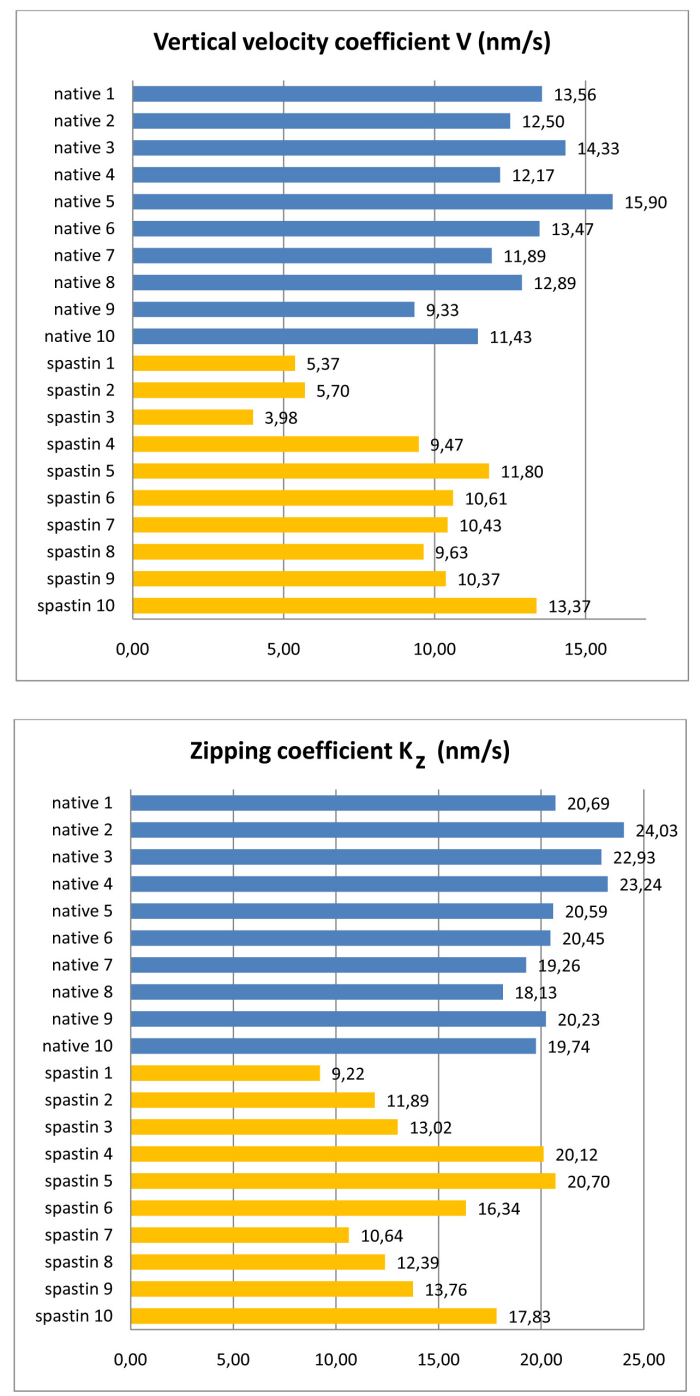

Figure 9: The height and zipping coefficients (in $\mathrm{nm} / \mathrm{s}$ ) of the model of Hutson et al. (2003) for the same ten native and ten modified (arm-gal4 x UAS-spastin-GFP) embryos for which our model's coefficients are shown in figure 6 . 

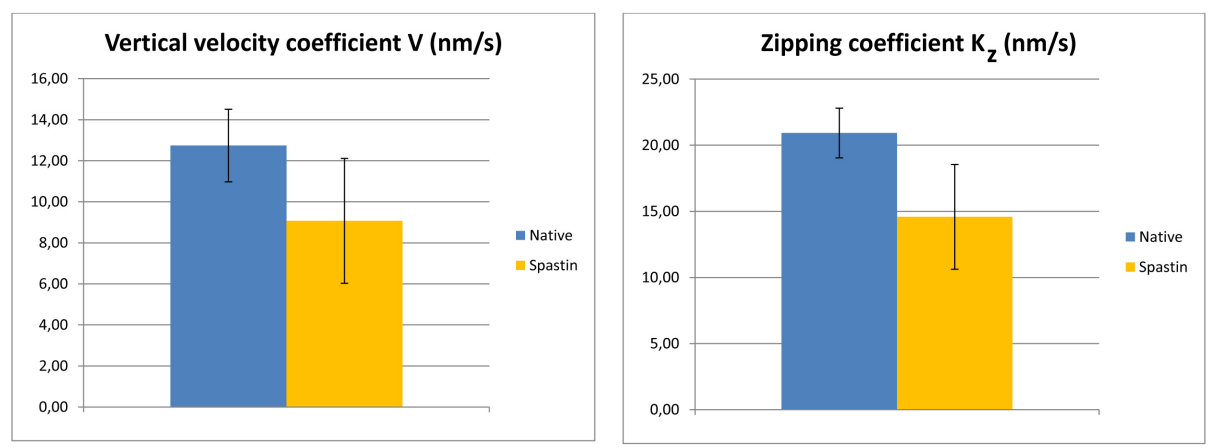

Figure 10: Average of the optimized height and zipping coefficients (in $\mathrm{nm} / \mathrm{s}$ ) of the model in Hutson et al. (2003) of ten native and ten modified (arm-gal4 x UASspastin-GFP) embryos presented in figure 9. Error bars represent the standard deviation of the data in each class.

the average zipping coefficients are downregulated in the Spastin setting but that the latter (which is the one we expect to be change significantly in the Spastin setting) is less well separated that in our model.

In table 5 we show the values of the cost function corresponding to the simulated contours using our model and using the previous model (Hutson et al. (2003)). For the model Hutson et al. (2003) we computed the values of $H(t)$ and $W(t)$ with (9) and (10), we reconstituted for each time step the two arcs of circle (symmetric relative to the dorso-ventral axis) to have the simulated dorsal opening and then, as before, we took the symmetric difference between it and the experimental dorsal opening to define the cost at that step. In order to correctly fit the model, at each time step we translated vertically the intersection of the two arcs of circle to place them on the straight line containing the experimental canthi. The cost function in the table 5 is expressed as average percentage (over the set of frames considered in the film being studied) of the area of the symmetric difference between the simulated and the experimental contour at each frame $i>1\left(\left|W_{i}^{E X P} \Delta W_{i}^{P D E}\right|\right.$, which, for us, is the simulation error at the previous time step) with respect to the area of the rectangular domain $M$ used in the simulations.

These values are shown just as an indication because although it is clear that our model yields significantly lower costs as indicated, this is not surprising since, unlike that of Hutson et al. (2003), it was specifically optimized to reduce the values of this cost function and moreover, being a PDE model and having less restrictions on the geometry (the two arcs of circle are not 


\begin{tabular}{|l|c|c|}
\hline Embryo & Our model & Model of [3] \\
\hline native 1 & $0.32 \%$ & $1.73 \%$ \\
native 2 & $0.31 \%$ & $1.54 \%$ \\
native 3 & $0.37 \%$ & $2.34 \%$ \\
native 4 & $0.46 \%$ & $2.44 \%$ \\
native 5 & $0.47 \%$ & $3.03 \%$ \\
native 6 & $0.53 \%$ & $3.54 \%$ \\
native 7 & $0.42 \%$ & $2.83 \%$ \\
native 8 & $0.40 \%$ & $2.22 \%$ \\
native 9 & $0.39 \%$ & $2.26 \%$ \\
native 10 & $0.36 \%$ & $2.01 \%$ \\
spastin 1 & $0.36 \%$ & $2.23 \%$ \\
spastin 2 & $0.48 \%$ & $1.79 \%$ \\
spastin 3 & $0.47 \%$ & $1.61 \%$ \\
spastin 4 & $0.63 \%$ & $2.49 \%$ \\
spastin 5 & $0.76 \%$ & $1.75 \%$ \\
spastin 6 & $0.54 \%$ & $1.69 \%$ \\
spastin 7 & $0.45 \%$ & $1.26 \%$ \\
spastin 8 & $0.29 \%$ & $0.72 \%$ \\
spastin 9 & $0.39 \%$ & $1.31 \%$ \\
spastin 10 & $0.46 \%$ & $1.10 \%$ \\
\hline
\end{tabular}

Table 1: The values of the cost function using our model and using the model of Hutson et al. (2003) of ten native and ten modified (arm-gal4 x UAS-spastin-GFP) embryos (the corresponding coefficients are shown in figure 6 for our model and in figure 9 for model of Hutson et al. (2003)). The cost function is the sum of the areas of the symmetric differences between experimental and simulated contours at each of the frames of the corresponding films. Here, it is expressed as an average percentage with respect to the area of the rectangular domain size used in the simulations. 
always a very good approximation of the actual shape), it has more flexibility to follow the actual contours and one more parameter.

\section{Conclusion}

The results obtained show very clearly that $C_{4}$, the zipping coefficient, is significantly downregulated in the Spastin setting: figure 7 shows a considerable decrease in this coefficient (on average). This is in agreement with the previously published results Jankovics and Brunner (2006). It is thus a good point for the pertinence of our model.

We notice that $C_{1}$, the coefficient associated with ectoderm resistance, is also downregulated. We were not looking for this effect but it is also consistent with what was described briefly in Jankovics and Brunner (2006) and with what we observe in our own movies. In fact, we can see that the cells in the lateral epidermis of our embryos (as well as those in the engrailed bands of the engrailed>spastin fly embryos in Jankovics and Brunner (2006)) are considerably less stretched in the dorso-ventral direction than those of the native embryos. Since Spastin affects microtubule organization, it could also affect the capacity of cells to generate and transmit forces. This might be an explanation for obtaining a lower value for $C_{1}$ in the Spastin setting.

Another point that is a good indication concerning our approach is the fact that inside each group, the coefficients measured for the different members are quite consistent - i.e. the dispersion of the data is relatively low (except for the last embryo in the Spastin group which seems to zip nearly like a native embryo). Moreover, the data presented in the previous section is just for 10 native embryos which are the natural control for the Spastin used. However, we also calculated these coefficients for several different native fly lines we have filmed for other purposes (different genotypes to express the GFP), and the values obtained were close to the ones shown here (data not shown).

To illustrate the precision that we can attain with the present model, we show in figures 11 and 12 (see also the supplementary material movie spastin.mov) the simulated contours given by our model in a Spastin embryo using the optimal constant force parameters we obtained. The results are less precise than in the native case. This is, in part, due to the more complex behavior of the canthi in the spastin setting. In fact, the zipping force reduction we obtain does not seem to be uniform along the boundary which allows some portions to zip before their neighbors on both sides 
yielding interior canthi and multiply connected holes (but his only happens very locally close to the canthi and at the end of DC, when the geometry is quite flat and the zipping is active in big portions of the boundary). It could be due to non-uniformity of our gal4 driver or other cell dependent factors influencing spastin's effect on filopodia. It is beyond the level of precision we aim for in this work (macroscopic, supracellular level) and is considered as a slight perturbation to the predicted behavior (yielding variability). Notice that level set methods are adapted to deal with this multiple connectivity of the domains (but less adapted to follow individual cells - when necessary we can use particle methods for that purpose).

Although a great part of the advantages of our model relative to the ODE model in Hutson et al. (2003) rely on its applicability to a wider variety of geometric situations which should be studied in future works, the comparison made in section 5 , shows that for this rather symmetric setting where the two models are applicable our model's zipping coefficient $\left(C_{4}\right)$ detects more clearly the dowregulation of the zipping in the Spastin setting that the previous model's zipping coefficient $\left(k_{z}\right)$. This is already a good indication of the added value provided by our approach - to detect weaker phenotypes it might be worthwhile to go through the significantly heavier computational effort of our model when compared to that of the previous one Hutson et al. (2003).

The results shown obtained in both the native and the Spastin setting are quite encouraging for using this model as a tool for identifying more subtle DC phenotypes than those that are accessible through traditional approaches. They should make it possible to single out new components of the DC process and give a better understanding the way they are put together to achieve such a precise and robust global behavior.

\section{Appendix A. Materials and Methods}

Genetics. The following fly lines were used: arm-gal4 (1560 from Bloomington Drosophila Stock Center), zipper-GFP (CC01226 from Flytrap Database), UAS-spastin-GFP (Trotta et al., 2004), ptc-gal4 (gift from N. Perrimon), en-gal4 (gift from A. Brand), UAS-actin5C-GFP (7310 from Bloomington Drosophila Stock Center) and UAS-D $\alpha$ catenin-GFP . The following recombined lines or crosses were used for video time-lapse of dorsal closure 1) $\mathrm{w}^{*}$,arm-gal4, zipper-GFP 2) $\mathrm{w}^{*}$, arm-gal4, zipper-GFP x UAS-spastin-GFP 3) $\mathrm{w}^{*}$, ptc-gal4, UAS-D $\alpha$ catenin-GFP 4) en-gal4 x UAS-actin5C-GFP. 

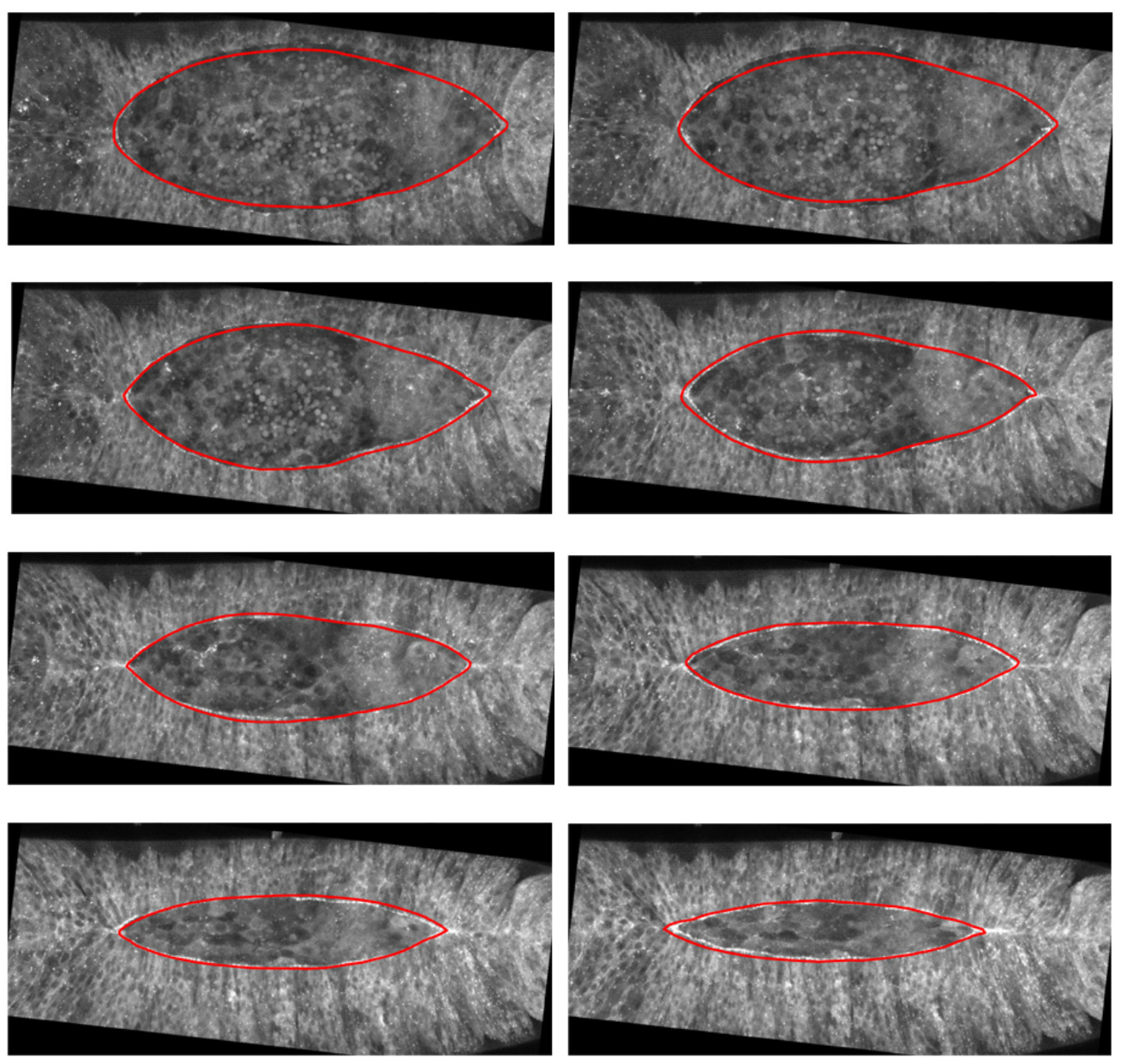

Figure 11: Dorsal Closure images at successive times with corresponding simulated contours (in red) for a Spastin embryo: first eight images of movie spastin.mov (genotype: arm-gal4,zipper-GFP x UAS-spastin-GFP). 

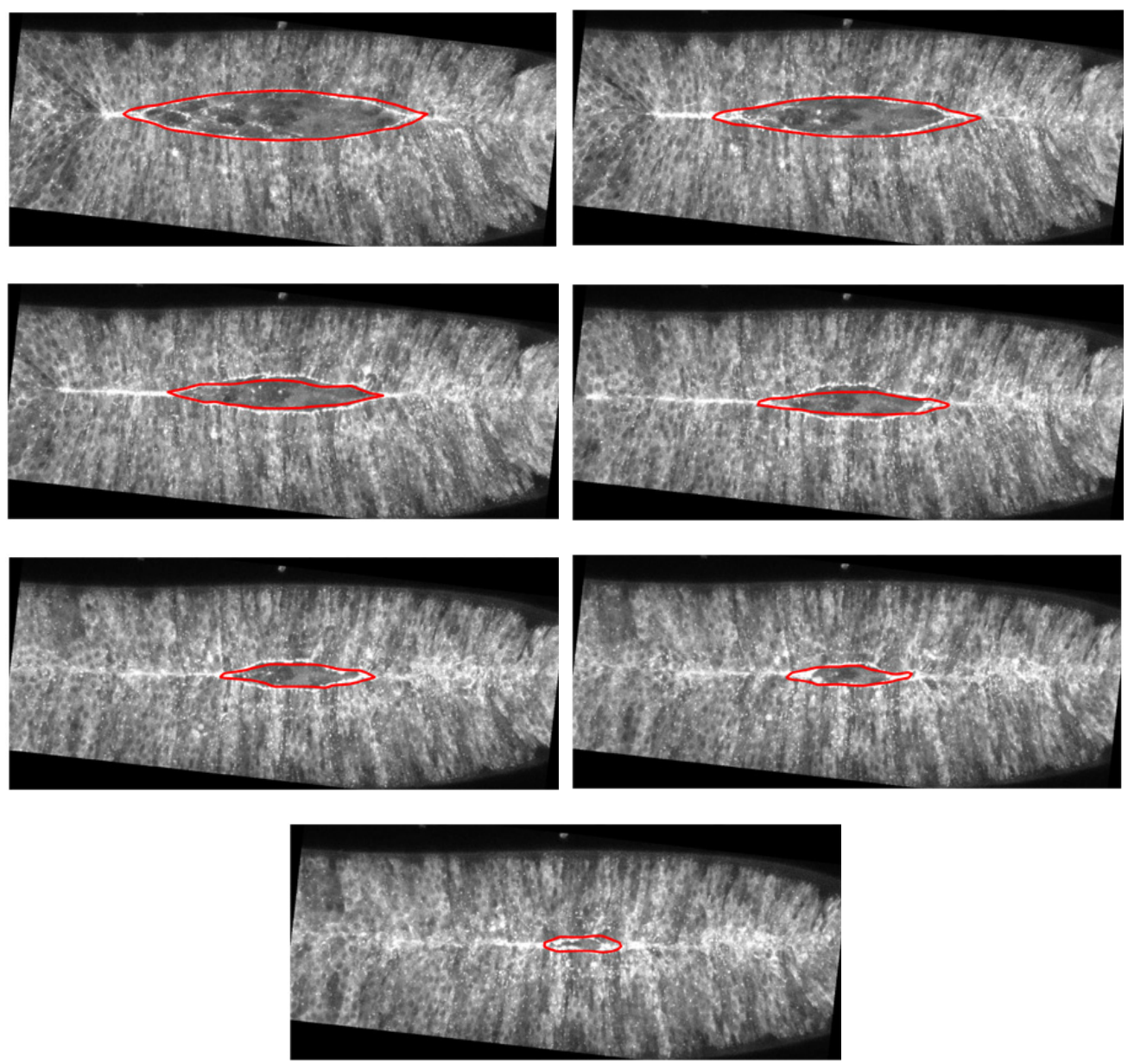

Figure 12: Dorsal Closure images at successive times with corresponding simulated contours (in red) for a Spastin embryo: next seven images of movie spastin.mov (genotype: arm-gal4,zipper-GFP x UAS-spastin-GFP). 
Live imaging and preliminary image treatment. Embryos are dechorionated in bleach then staged and placed dorsal side down on a coverslip. A coating of halocarbon oil, a piece of damp paper and a hermetic chamber ensure hydration (and normal development of $95 \%$ of embryos into larvae in control experiments). Embryos are neither fixed, pressed, nor glued, to ensure that the dynamics is modified as little as possible by external forces. They naturally lay on their dorsal part when put in the viscous oil, which is convenient to film dorsal closure. This method is sufficient to immobilize the external part of the organism but movements are still possible inside the vitelline membrane (the membrane is a protective layer outside the epidermis - larvae hatch out of it after embryogenesis). Images are taken with a Zeiss LSM 510 Meta confocal inverted microscope using x40 1.3 NA oil immersion objectives. Films last from 2 to 3 hours with stacks of 25 images (thickness from 30 to $40 \mu \mathrm{m}$ ) per time point. We assemble images and movies using ImageJ. Stacks are projected using a maximal intensity projection.

\section{Appendix B. Movie Captions}

native.mov Confocal time-lapse images of a $\mathrm{w}^{*}$,arm-gal4, zipper-GFP embryo, which has native closure dynamics (these are the control embryos we use in this work).

zip.mov: Confocal time-lapse images of a en-gal4, UAS-actin5C-GFP embryo during DC. Actin filopodia are visible in the posterior half part of the segments (actin-GFP expressed under the control of engrailed). Note that the speed is twice slower in the second part of the movie, when a zoom is made on the central segments. en-gal4 (gift from A. Brand), UAS-actin5C-GFP (7310 from Bloomington Drosophila Stock Center).

track.mov: Confocal time-lapse images taken from an embryo showing in gray a membrane marker only in the anterior compartments. Parasegmental and segmental boundaries can thus be tracked during the closure. Note that some cells in posterior compartments (see arrowheads on the first image) are marked too but the segmental boundary lies just posterior to these cells. (Genotype: ptc-gal4, UAS-D $\alpha$ catenin-GFP).

spastin.mov: Confocal time-lapse images of a $\mathrm{w}^{*}$, arm-gal4, zipper-GFP x UAS-spastin-GFP embryo during DC (this is the movie used for making the simulations presented in figures 11 and 12). 


\section{Appendix C. Acknowledgments}

This project was supported by the ACI NIM Momatsouti. Work in the SN laboratory is supported by CNRS, ANR, ARC, FRM, CEFIPRA.

Allaire, G., 2007. Conception optimale de structures. Vol. 58 of Mathématiques \& Applications (Berlin) [Mathematics \& Applications]. Springer-Verlag, Berlin, with the collaboration of Marc Schoenauer (INRIA) in the writing of Chapter 8.

Almeida, L., Bagnerini, P., Habbal, A., Noselli, S., Serman, F., 2009, http://hal.archives-ouvertes.fr/hal-00480769/en/. Tissue repair modeling. In: Novaga, M., Orlandi, G. (Eds.), Singularities in Nonlinear Evolution Phenomena and Applications. Scuola Normale Superiore, pp. 27-46.

URL http://hal .archives-ouvertes.fr/hal-00480769/en/

Gettings, M., Serman, F., Rousset, R., Bagnerini, P., Almeida, L., Noselli, S., 2010. Jnk signalling controls remodelling of the segment boundary through cell reprogramming during drosophila morphogenesis. PLoS Biol 8 (6), e1000390.

URL http://dx.doi.org/10.1371/journal.pbio.1000390

Hutson, M. S., Tokutake, Y., Chang, M.-S., Bloor, J. W., Venakides, S., Kiehart, D. P., Edwards, G. S., Apr 2003. Forces for morphogenesis investigated with laser microsurgery and quantitative modeling. Science 300 (5616), 145-149.

URL http://dx.doi.org/10.1126/science.1079552

Jacinto, A., Wood, W., Balayo, T., Turmaine, M., Martinez-Arias, A., Martin, P., Nov 2000. Dynamic actin-based epithelial adhesion and cell matching during drosophila dorsal closure. Curr Biol 10 (22), 1420-1426.

Jankovics, F., Brunner, D., Sep 2006. Transiently reorganized microtubules are essential for zippering during dorsal closure in drosophila melanogaster. Dev Cell 11 (3), 375-385.

URL http://dx.doi.org/10.1016/j.devcel.2006.07.014

Kiehart, D. P., Galbraith, C. G., Edwards, K. A., Rickoll, W. L., Montague, R. A., Apr 2000. Multiple forces contribute to cell sheet morphogenesis for dorsal closure in drosophila. J Cell Biol 149 (2), 471-490. 
Kimmel, R., 2004. Numerical geometry of images. Springer-Verlag, New York.

Layton, A. T., Toyama, Y., Yang, G.-Q., Edwards, G. S., Kiehart, D. P., Venakides, S., Dec 2009. Drosophila morphogenesis: tissue force laws and the modeling of dorsal closure. HFSP J 3 (6), 441-460.

URL http://dx.doi.org/10.2976/1.3266062

Ma, X., Lynch, H. E., Scully, P. C., Hutson, M. S., 2009. Probing embryonic tissue mechanics with laser hole drilling. Phys Biol 6 (3), 036004.

URL http://dx.doi.org/10.1088/1478-3975/6/3/036004

Martin, A. C., Kaschube, M., Wieschaus, E. F., Jan 2009. Pulsed contractions of an actin-myosin network drive apical constriction. Nature 457 (7228), 495-499.

URL http://dx.doi.org/10.1038/nature07522

Millard, T. H., Martin, P., Feb 2008. Dynamic analysis of filopodial interactions during the zippering phase of drosophila dorsal closure. Development 135 (4), 621-626.

URL http://dx.doi.org/10.1242/dev.014001

Mitchell, I. M., 2008. The flexible, extensible and efficient toolbox of level set methods. J. Sci. Comput. 35 (2-3), 300-329.

Murray, J. D., 2003. On the mechanochemical theory of biological pattern formation with application to vasculogenesis. Comptes Rendus Biologies 326 (2), $239-252$.

URL http://www.sciencedirect.com/science/article/B6X1F-4893WR0-7/2/c8300e5b2f07

Olsen, L., Maini, P. K., Sherratt, J. A., 1998. Spatially varying equilibria of mechanical models: application to dermal wound contraction. Math. Biosci. 147 (1), 113-129.

URL http://dx.doi.org/10.1016/S0025-5564(97)00075-8

Olsen, L., Sherratt, J. A., Maini, P. K., 1995. A mechanochemical model for adult dermal wound contraction and the permanence of the contracted tissue displacement profile. Journal of Theoretical Biology 177 (2), 113 - 128.

URL http://www . sciencedirect.com/science/article/B6WMD-45R8FPF-F/2/8bd33dd039cb 
Osher, S., Fedkiw, R., 2003. Level set methods and dynamic implicit surfaces. Vol. 153 of Applied Mathematical Sciences. Springer-Verlag, New York.

Osher, S., Sethian, J. A., 1988. Fronts propagating with curvature-dependent speed: Algorithms based on hamilton-jacobi formulations. J. Comput. Phys. 79 (1), 12-49.

Osher, S., Shu, C.-W., 1991. High-order essentially nonoscillatory schemes for hamilton-jacobi equations. SIAM J. Numer. Anal. 28 (4), 907-922.

Peralta, X. G., Toyama, Y., Hutson, M. S., Montague, R., Venakides, S., Kiehart, D. P., Edwards, G. S., Apr 2007. Upregulation of forces and morphogenic asymmetries in dorsal closure during drosophila development. Biophys J 92 (7), 2583-2596.

URL http://dx.doi.org/10.1529/biophysj.106.094110

Quarteroni, A., 2009. Numerical models for differential problems. Vol. 2 of MS\&A. Modeling, Simulation and Applications. Springer-Verlag Italia, Milan.

Sethian, J. A., 1999. Level set methods and fast marching methods, 2nd Edition. Vol. 3 of Cambridge Monographs on Applied and Computational Mathematics. Cambridge University Press, Cambridge.

Solon, J., Kaya-Copur, A., Colombelli, J., Brunner, D., Jun 2009. Pulsed forces timed by a ratchet-like mechanism drive directed tissue movement during dorsal closure. Cell 137 (7), 1331-1342.

URL http://dx.doi.org/10.1016/j.cell.2009.03.050 